\title{
Histone deacetylases 1, 6 and 8 are critical for invasion in breast cancer
}

\author{
SOON YOUNG PARK* ${ }^{*}$ JI AE JUN* ${ }^{*}$ KANG JIN JEONG, HOI JEONG HEO, JANG SIHN SOHN, \\ HOI YOUNG LEE, CHANG GYO PARK and JAEKU KANG
}

Myunggok Medical Research Institute, College of Medicine, Konyang University, Daejeon 302-718, Republic of Korea

Received January 7, 2011; Accepted March 3, 2011

DOI: 10.3892/or.2011.1236

\begin{abstract}
Histone deacetylases (HDACs) are associated with the development and progression of cancer, but it is not known which of the HDAC isoforms play important roles in breast cancer metastasis. This study identified the specific HDAC isoforms that are necessary for invasion and/or migration in human breast cancer cell lines. MDA-MB-231 cells were significantly more invasive and expressed higher levels of matrix metalloproteinase-9 (MMP-9) compared to MCF-7 cells. We compared the expression of HDAC isoforms between MCF-7 and MDA-MB-231 cells and found greater expression of HDAC4, 6 and 8 in MDA-MB-231 cells by RT-PCR and Western blot analyses. In addition, apicidin, a histone deacetylase inhibitor, was shown to attenuate the invasion, migration and MMP-9 expression in MDA-MB-231 cells. Using specific siRNAs directed against HDAC1, 4, 6 and 8, we show that inhibition of HDAC1, 6 and 8, but not HDAC4, are responsible for invasion and MMP-9 expression in MDA-MB-231 cells. We analyzed the invasiveness of MCF-7 cells overexpressing HDAC1, 4, 6 or 8 and found that overexpression of HDAC1, 6 or 8 increased invasion and MMP-9 expression. By developing HDAC isoforms as potential biomarkers for breast cancer metastasis, the present study can be extended to developing therapies for breast cancer invasion.
\end{abstract}

\section{Introduction}

Histone deacetylases (HDACs) are emerging as critical regulators of cell growth, differentiation and apoptosis. HDAC activity in human tumors leads to conformational changes within the nucleosome, which results in the transcriptional

Correspondence to: Dr Chang Gyo Park or Dr Jaeku Kang, Department of Pharmacology, College of Medicine, Konyang University, Daejeon 302-718, Republic of Korea

E-mail: aruso@konyang.ac.kr

E-mail: jaeku@konyang.ac.kr

*Contributed equally

Key words: histone deacetylases, HDAC isoforms, MMP-9, invasion, breast cancer cells repression of genes during migration and metastasis (1). Changes in HDAC expression levels have been associated with clinical prognosis in patients with invasive cancer, including breast cancer (2,3). HDAC1 (4-6), HDAC2 $(7,8)$ and HDAC3 (5) have been found to be expressed at higher levels in cancer tissues. Proliferation of lung, colon and cervical cancer cell lines is reduced after HDAC8 knockdown (9), and HDAC6 mediates estrogen-induced increase in cell motility (10). However, the molecular mechanisms underlying the HDAC isoform functions during invasion remain unclear.

Cancer invasion and metastasis are the leading causes of mortality in patients with breast cancer. Tumor metastasis consists of cell adhesion, invasion and angiogenesis (11). Matrix metalloproteinases (MMPs) have been recognized as important mediators of extracellular matrix (ECM) degradation (12). MMPs and MMP inhibitors have been extensively investigated in human breast cancer clinical studies $(13,14)$. It has been reported that MMP-2 has a role in the H-ras-induced invasive phenotype of MCF10A human breast epithelial cells (15), analogous to the role of MMP-9 expression in the metastatic phenotype of transformed rat embryo cells (16).

This study was aimed at determining the role of HDACdependent invasion and migration in breast cancer and examining the HDAC-dependent regulation of MMP-9 expression in breast cancer cell migration and invasion.

\section{Materials and methods}

Cell culture. Human breast cancer MDA-MB-231 and MCF-7 cells were obtained from ATCC (Manassas, VA). Cells were maintained in RPMI-1640 medium supplemented with $10 \%$ fetal bovine serum and incubated in a humidified atmosphere containing $5 \% \mathrm{CO}_{2}$ at $37^{\circ} \mathrm{C}$.

Reagents. Apicidin was obtained from Sigma (St. Louis, MO). Antibodies against HDAC1 and 8 were purchased from Millipore (Billerica, MA). Antibodies against HDAC2, 3 and 4 were obtained from Abcam (Cambridge, MA). Antibodies against HDAC6 and glyceraldehyde-3-phosphate dehydrogenase (GAPDH) were purchased from Santa Cruz Biotechnology (Santa Cruz, CA).

Reverse transcription-PCR. Total cellular RNA $(1 \mu \mathrm{g})$ isolated from cultured cell lines was used for reverse transcription. The 
cDNA was then amplified with primer sets for HDAC1, 2, 3, 4, 6, 8, MMP-9, 2 and GAPDH. Primer sequences are as follows: HDAC1 forward, 5'-GGAAATCTATCGCCCTCACA-3' and reverse, 5'-TTGCCACAGAACCACCAGTA-3'; HDAC2 forward, 5'-ATAAAGCCACTGCCGAAGAA-3' and reverse, 5'-TCCTCCAGCCCAATTAACAG-3'; HDAC3 forward, 5'-ACGTGGGCAACTTCCACTAC-3' and reverse, 5'- GACTCTTGGTGAAGCCTTGC-3'; HDAC4 forward, 5'-AGCGTGAGCAAGATCCTCAT-3' and reverse, 5'-GCCAAGTACTCAGCGTCTCC-3'; HDAC6 forward, 5'-TATCTGCCCCAGTACCTTCG-3' and reverse, 5'-GGACATCCCAATCCACAATC-3'; HDAC8 forward, 5'-GGTGACGTGTCTGATGTTGG-3' and reverse, 5'-GACACTTGCCAATTCCCACT-3'; GAPDH forward, 5'-CATCT TCCAGGAGCGAGA-3' and reverse, 5'-CTGCTTCACCACCTTCTTGAT-3'; MMP-9 forward, 5'-CATCGTCATCCAGTTTGG-3' and reverse, 5'-GATGGATTGGCCTTGGAA-3' and MMP-2 forward, 5'-GGCCTCTCCTGACATTGACCTT-3' and reverse, 5'-GGC CTCGTATACCGCATCAATC-3'.

Western blotting. Cells were plated in 6-well plates and treated with apicidin for $24 \mathrm{~h}$. After treatment, cells were harvested and washed twice with PBS at $4^{\circ} \mathrm{C}$. Total proteins were prepared by cell lysis in $200 \mu 1$ of ice-cold RIPA buffer, and lysates were resolved by 10 or $12 \%$ SDS-PAGE gels and transferred to PVDF membranes. The membranes were blocked with blocking buffer containing $5 \%$ non-fat dry milk for $1 \mathrm{~h}$ at room temperature and incubated overnight at $4^{\circ} \mathrm{C}$ with the antibodies indicated. After washing for $1 \mathrm{~h}$ with TBS containing $0.1 \%$ Tween-20, membranes were incubated with horseradish peroxidase-conjugated anti-rabbit or anti-goat antibodies for $1 \mathrm{~h}$. After washing three times with TBS containing $0.1 \%$ Tween-20, the immunoreactive bands were visualized using ECL.

Small interfering RNA (siRNA) and plasmid transfection. Specific siRNAs for HDAC1, 6, 8 (sc-29343, sc-35544, sc-35548, Santa Cruz Biotechnology) and HDAC4 (J-00349707-0005, Dharmacon, Lafayette, CO) or a scrambled siRNA (Invitrogen, Carlsbad, CA) were obtained. HDAC1 and HDAC4 constructs were kindly provided by Dr S.L. Schreiber (Howard Hughes Medical Institute, Department of Chemistry and Chemical Biology, Harvard University, Cambridge, MA) and Dr E. Seto (Moffitt Cancer Center, University of South Florida, Tampa, FL), respectively. HDAC6 and HDAC8 constructs were purchased from Addgene (Cambridge, MA). Cells were transfected with Lipofectamine 2000 (Invitrogen) according to the manufacturer's instructions.

Invasion assay. Invasion assays were performed in triplicate, using a 48 -well microchemotaxis chamber with $8-\mu \mathrm{m}$-pore membranes (Neuroprobe) pre-coated with $10 \mu \mathrm{g} / \mathrm{ml}$ Matrigel (BD Bioscience). The bottom chamber was filled with $1 \%$ FBS/RPMI (MDA-MB-231) or 20\% FBS/RPMI (MCF-7), and then cells that had been treated with apicidin for $24 \mathrm{~h}$ were placed in the upper chamber and incubated at $37^{\circ} \mathrm{C}$ for 24 h. The membranes were fixed and stained with Diff-Quik reagents (Dade Behring, Inc., Deerfield, IL).

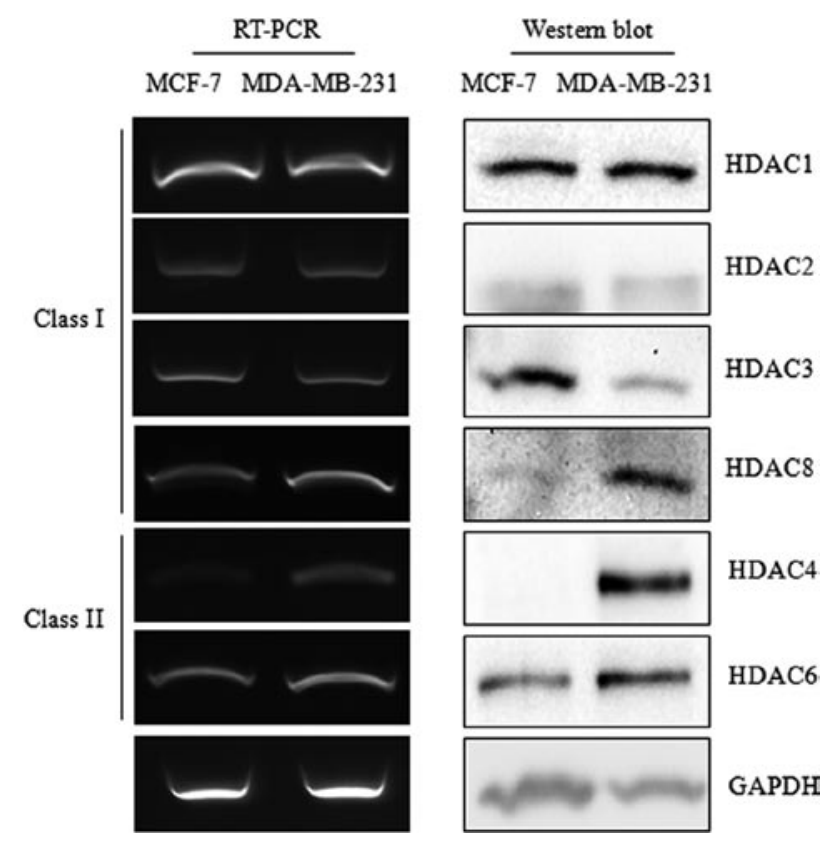

Figure 1. Expression of HDACs in breast cancer cells. Total RNA and cell lysates were prepared from MCF-7 and MDA-MB-231 cells. The levels of mRNA and protein were determined by RT-PCR (left) and Western blotting (right), respectively.

Wound healing assay. Cells were grown to confluence in 6-well plates and serum starved overnight. Cells were scraped with a P200 pipette tip and treated with apicidin for $24 \mathrm{~h}$. Migration of cells into the wound was examined by phase contrast microscopy. Photographic images were obtained immediately after scraping and after $24 \mathrm{~h}$ in the same locations.

Statistical analysis. Results are expressed as the mean \pm standard deviation (SD), and an analysis was performed by a one-way Student's t-test.

\section{Results}

Apicidin inhibits invasion and migration in breast cancer cells. We first compared the expression of HDAC isoforms in two cell lines, MDA-MB-231 (a highly metastatic breast cancer cell line) and MCF-7 (a less invasive breast cancer cell line). HDAC1 and HDAC2 expression patterns were similar in both cell lines. However, the expression of HDAC4, 6 and 8 was higher in MDA-MB-231 cells compared to MCF-7 cells (Fig. 1).

We next examined whether apicidin, an inhibitor of histone deacetylases, inhibited invasion and migration of MDA-MB231 cells. As shown in Fig. 2A and B, MDA-MB-231 cells exhibited greater invasion and migration than MCF-7 cells. Apicidin treatment of MDA-MB-231 cells inhibited invasion and migration in a dose-dependent manner.

Apicidin has been reported to inhibit MMP-2 activity more than MMP-9 in human breast epithelial cells (17). To test the effect of apicidin on MMP activity, we treated cells with increasing amounts of apicidin and observed mRNA expression using RT-PCR. Apicidin inhibited MMP-9 expression in MDA-MB-231 cells (Fig. 2C) but did not inhibit MMP-2 activity (data not shown). 
$\mathbf{A}$

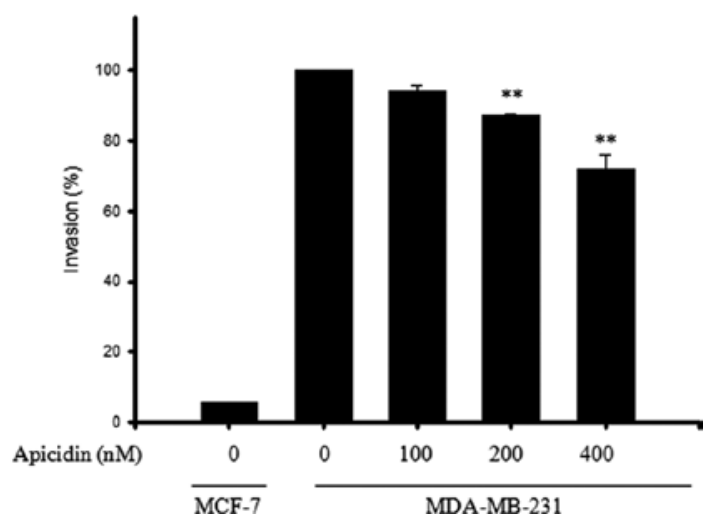

B

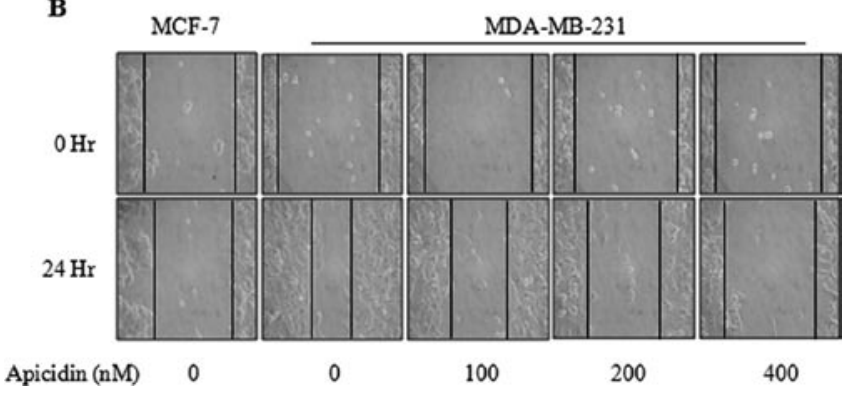

C

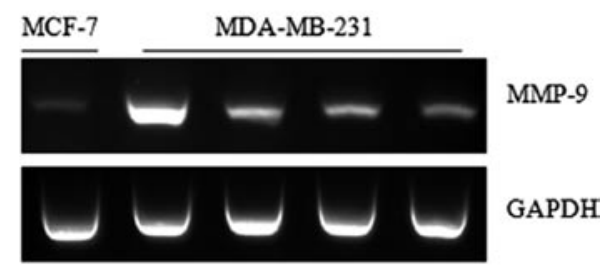

$\begin{array}{llllll}\text { Apicidin (nM) } & 0 & 0 & 100 & 200 & 400\end{array}$

Figure 2. Apicidin inhibits invasion and migration in breast cancer cells. (A and B) Cells were treated with the indicated concentrations of apicidin for $24 \mathrm{~h}$ prior to an invasion assay (A) or a wound healing assay (B). (C) Cells were serum starved for $24 \mathrm{~h}$ and then treated with the indicated concentrations of apicidin for $24 \mathrm{~h}$. Expression of mRNA was then determined by RT-PCR. Results show the mean \pm standard deviation (SD) of three independent experiments performed in triplicate. ${ }^{* *} \mathrm{p}<0.01$ vs. control.

Specific HDACs involved in invasion and MMP-9 expression. The comparison of HDAC expression between MDA-MB-231 and MCF-7 cells indicated that high expression of HDAC4, 6 and 8 was significantly associated with invasion. In addition, HDAC1 has been previously demonstrated to be associated with invasion (18). Based on these criteria, HDAC1, 4, 6 and 8 were selected for further study. We used specific HDAC siRNA to determine which HDACs were involved in invasion. Treatment of MDA-MB-231 cells with HDAC1, 6 and 8 siRNAs inhibited invasion (Fig. 3A). Each specific siRNA caused a marked decrease in specific mRNA levels without affecting the mRNA levels of the other HDACs. The knockdown of HDAC1, 6 and 8 caused decrease of MMP-9 mRNA expression (Fig. 3B). However, HDAC4 knockdown did not affect invasion or MMP-9 expression.
A

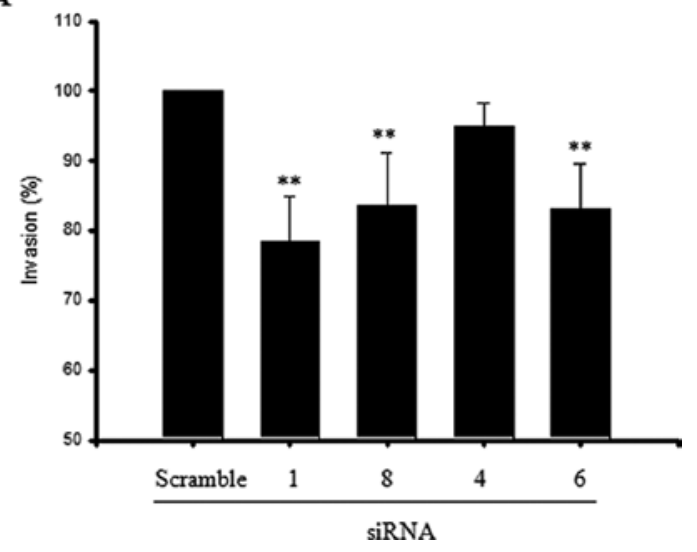

B

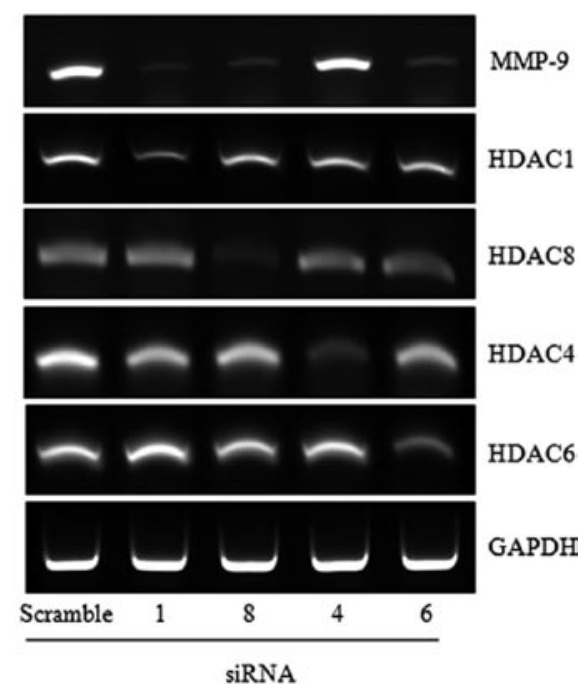

Figure 3. Specific HDAC siRNA decreases invasion and MMP-9 expression. MDA-MB-231 cells were transfected with either scrambled or specific HDAC siRNA for $48 \mathrm{~h}$, followed by an invasion assay (A) and RT-PCR (B). Results show the mean \pm standard deviation (SD) of three independent experiments performed in triplicate. ${ }^{* *} \mathrm{p}<0.01$ vs. control.

To confirm the effects of HDACs on cell invasion, we established MCF-7 cells that overexpress HDAC1, 4, 6 or 8 DNA constructs by transient transfection. After $48 \mathrm{~h}$, we performed invasion assays and found that the overexpression of HDAC1, 6 and 8 in MCF-7 cells resulted in increased invasion (Fig. 4A). More importantly, overexpression of these HDAC isoforms in MCF-7 cells also increased MMP-9 mRNA expression (Fig. 4B).

\section{Discussion}

In this study, we examined whether HDAC isoform status plays a role in the differential responses of MCF-7 and MDA-MB231 cells. Many reports have correlated HDAC expression levels with clinical prognosis in patients with invasive cancers, including breast cancer $(2,3,6,10,19)$. This study is the first to investigate the expression of various HDAC isoforms. It has been proposed that HDAC expression leads to increased MMP expression and cell invasion. Apicidin prevents an H-rasinduced invasive phenotype (17). Our data suggest that treating 
A

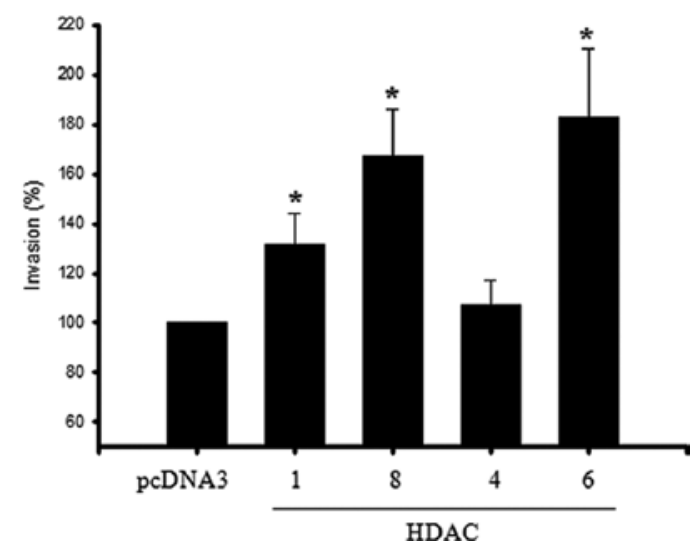

B

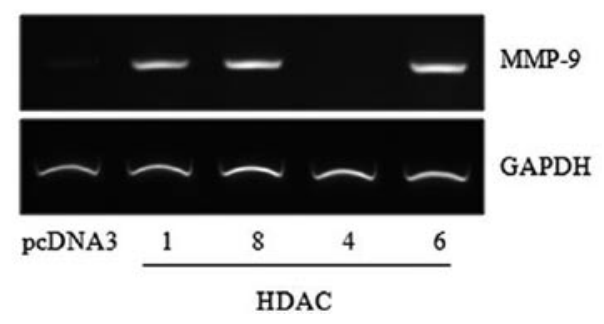

Figure 4. HDAC1, 6 and 8 are involved in invasion and MMP-9 expression. MCF-7 cells were transfected with pcDNA3 (vector) or HDAC1, 4, 6 or 8 for $48 \mathrm{~h}$, followed by an invasion assay (A) and RT-PCR (B). Results show the mean \pm standard deviation (SD) of three independent experiments performed in triplicate. ${ }^{*} \mathrm{p}<0.05$ vs. control.

MDA-MB-231 cells with apicidin inhibits invasion, migration and MMP-9 expression in a dose-dependent manner.

HER-2/neu inhibits the metastasis suppressor RECK via an Sp1- and HDAC1-dependent mechanism, to promote cell invasion (20). In addition, HDAC1 has been found to be involved in the migration and invasion of cancer cells (18). In this study, HDAC1 expression was similar in both MDA-MB231 and MCF-7 cell lines but was also found to be important for invasion. Cell lines representative of ER-positive (MCF-7) and ER-negative (MDA-MB-231) human breast cancer cells can both differentiate to invasion. An ER-negative and invasive human breast cancer cell line, MDA-MB-231, has constitutively higher mitogen-activated protein kinase (ERK1\&2/ MAPK) when compared to the ER-positive and non-invasive MCF-7 (21). Thus, the importance of HDAC1 in breast cancer cells will be the topic of future investigations.

We found that MDA-MB-231 cells express higher levels of HDAC4, 6 and 8 than MCF-7 cells. Specific knockdown of HDAC isoforms in MDA-MB-231 cells (silencing of HDAC1, 6 and 8) abolished invasion and MMP-9 expression. Reconstitution of HDAC1, 4, 6 and 8 in MCF-7 cells, as well as overexpression of HDAC1, 6 and 8 but not 4, increased invasion and MMP-9 expression. HDAC4 is a key mediator of p53-dependent cancer cell growth arrest in response to DNA damage (22-24); therefore, we propose that HDAC4 acts as an anti-proliferative and a pro-survival factor for cancer cells.
Our data suggest that inhibitor and siRNA of HDACs abolished breast cancer invasion by regulating the expression of factors involved in the degradation of the ECM. MMP-2, 9, 11, 13 and 14 are known to enhance the invasiveness of MDA-MB-231 cells $(25,26)$. HDACs are known to regulate the expression of MMPs $(27,28)$. Therefore, the identification of cancer-related HDAC isoforms has therapeutic interest in oncology for more specific HDAC inhibitors with fewer side effects for patients.

In conclusion, we have examined the functional role of HDACs in invasion according to their expression in MCF-7 and MDA-MB-231 cells. Our findings implicate HDAC1, 6 and 8 in the progression of breast cancer cells.

\section{Acknowledgements}

This study was supported by the Basic Science Research Program through the National Research Foundation of Korea (NRF), funded by the Ministry of Education, Science and Technology (2009-0063816) and, in part, by the Konyang University Myunggok Research Fund of 2010-12.

\section{References}

1. Minucci S and Pelicci PG: Histone deacetylase inhibitors and the promise of epigenetic (and more) treatments for cancer. Nat Rev 6: 38-51, 2006.

2. Krusche CA, Wulfing $\mathrm{P}$, Kersting C, Vloet A, Bocker W, Kiesel L, Beier HM and Alfer J: Histone deacetylase-1 and -3 protein expression in human breast cancer: a tissue microarray analysis. Breast Cancer Res Treat 90: 15-23, 2005.

3. Zhang Z, Yamashita H, Toyama T, Sugiura H, Omoto Y, Ando Y, Mita K, Hamaguchi M, Hayashi S and Iwase H: HDAC6 expression is correlated with better survival in breast cancer. Clin Cancer Res 10: 6962-6968, 2004.

4. Zhang Z, Yamashita H, Toyama T, Sugiura H, Ando Y, Mita K, Hamaguchi M, Hara Y, Kobayashi S and Iwase H: Quantitation of HDAC1 mRNA expression in invasive carcinoma of the breast. Breast Cancer Res Treat 94: 11-16, 2005.

5. Wilson AJ, Byun DS, Popova N, Murray LB, L'Italien K, Sowa Y, Arango D, Velcich A, Augenlicht LH and Mariadason JM: Histone deacetylase 3 (HDAC3) and other class I HDACs regulate colon cell maturation and p21 expression and are deregulated in human colon cancer. J Biol Chem 281: 13548-13558, 2006.

6. Choi JH, Kwon HJ, Yoon BI, Kim JH, Han SU, Joo HJ and Kim DY: Expression profile of histone deacetylase 1 in gastric cancer tissues. Jpn J Cancer Res 92: 1300-1304, 2001.

7. Zhu P, Martin E, Mengwasser J, Schlag P, Janssen KP and Gottlicher M: Induction of HDAC2 expression upon loss of APC in colorectal tumorigenesis. Cancer Cell 5: 455-463, 2004.

8. Huang BH, Laban M, Leung CH, Lee L, Lee CK, Salto-Tellez M, Raju GC and Hooi SC: Inhibition of histone deacetylase 2 increases apoptosis and p21Cip1/WAF1 expression, independent of histone deacetylase 1. Cell Death Differ 12: 395-404, 2005.

9. Vannini A, Volpari C, Filocamo G, Casavola EC, Brunetti M, Renzoni D, Chakravarty P, Paolini C, De Francesco R, Gallinari P, Steinkuhler C and Di Marco S: Crystal structure of a eukaryotic zinc-dependent histone deacetylase, human HDAC8, complexed with a hydroxamic acid inhibitor. Proc Natl Acad Sci USA 101: 15064-15069, 2004.

10. Saji S, Kawakami M, Hayashi S, Yoshida N, Hirose M, Horiguchi S, Itoh A, Funata N, Schreiber SL, Yoshida M and Toi M: Significance of HDAC6 regulation via estrogen signaling for cell motility and prognosis in estrogen receptor-positive breast cancer. Oncogene 24: 4531-4539, 2005.

11. Cavallaro U and Christofori G: Cell adhesion in tumor invasion and metastasis: loss of the glue is not enough. Biochim Biophys Acta 1552: 39-45, 2001.

12. Eiseler T, Doppler H, Yan IK, Kitatani K, Mizuno K and Storz P: Protein kinase D1 regulates cofilin-mediated F-actin reorganization and cell motility through slingshot. Nature cell biology 11: 545-556, 2009. 
13. Duffy MJ, Maguire TM, Hill A, McDermott E and O'Higgins $\mathrm{N}$ : Metalloproteinases: role in breast carcinogenesis, invasion and metastasis. Breast Cancer Res 2: 252-257, 2000.

14. Zhang B, Cao X, Liu Y, Cao W, Zhang F, Zhang S, Li H, Ning L, Fu L, Niu Y, Niu R, Sun B and Hao X: Tumor-derived matrix metalloproteinase-13 (MMP-13) correlates with poor prognoses of invasive breast cancer. BMC Cancer 8: 83-92, 2008.

15. Moon A, Kim MS, Kim TG, Kim SH, Kim HE, Chen YQ and Kim HR: H-ras, but not N-ras, induces an invasive phenotype in human breast epithelial cells: a role for MMP-2 in the H-rasinduced invasive phenotype. Int J Cancer 85: 176-181, 2000.

16. Bernhard EJ, Gruber SB and Muschel RJ: Direct evidence linking expression of matrix metalloproteinase 9 (92-kDa gelatinase/ collagenase) to the metastatic phenotype in transformed rat embryo cells. Proc Natl Acad Sci USA 91: 4293-4297, 1994.

17. Kim MS, Son MW, Kim WB, In Park Y and Moon A: Apicidin an inhibitor of histone deacetylase, prevents H-ras-induced invasive phenotype. Cancer Lett 157: 23-30, 2000.

18. Jeon HW and Lee YM: Inhibition of histone deacetylase attenuates hypoxia-induced migration and invasion of cancer cells via the restoration of RECK expression. Mol Cancer Ther 9: 1361-1370, 2010

19. Ishihama K, Yamakawa M, Semba S, Takeda H, Kawata S, Kimura S and Kimura W: Expression of HDAC1 and CBP/p300 in human colorectal carcinomas. J Clin Pathol 60: 1205-1210, 2007.

20. Hsu MC, Chang HC and Hung WC: HER-2/neu represses the metastasis suppressor RECK via ERK and Sp transcription factors to promote cell invasion. J Biol Chem 281: 4718-4725, 2006.

21. Krueger JS, Keshamouni VG, Atanaskova N and Reddy KB Temporal and quantitative regulation of mitogen-activated protein kinase (MAPK) modulates cell motility and invasion. Oncogene 20: 4209-4218, 2001.
22. Kao GD, McKenna WG, Guenther MG, Muschel RJ, Lazar MA and Yen TJ: Histone deacetylase 4 interacts with 53BP1 to mediate the DNA damage response. J Cell Biol 160: 1017-1027, 2003.

23. Imbriano C, Gurtner A, Cocchiarella F, Di Agostino S, Basile V, Gostissa M, Dobbelstein M, Del Sal G, Piaggio G and Mantovani R: Direct p53 transcriptional repression: in vivo analysis of CCAAT-containing G2/M promoters. Mol Cell Biol 25: 3737-3751, 2005.

24. Basile V, Mantovani R and Imbriano C: DNA damage promotes histone deacetylase 4 nuclear localization and repression of G2/M promoters, via p53 C-terminal lysines. J Biol Chem 281: 2347-2357, 2006

25. Hegedus L, Cho H, Xie X and Eliceiri GL: Additional MDA-MB231 breast cancer cell matrix metalloproteinases promote invasiveness. J Cell Physiol 216: 480-485, 2008.

26. Ramos-DeSimone N, Hahn-Dantona E, Sipley J, Nagase H, French DL and Quigley JP: Activation of matrix metalloproteinase-9 (MMP-9) via a converging plasmin/stromelysin-1 cascade enhances tumor cell invasion. J Biol Chem 274: 13066-13076, 1999.

27. Liu LT, Chang HC, Chiang LC and Hung WC: Histone deacetylase inhibitor up-regulates RECK to inhibit MMP-2 activation and cancer cell invasion. Cancer Res 63: 3069-3072, 2003.

28. Klampfer L, Huang J, Shirasawa S, Sasazuki T and Augenlicht L: Histone deacetylase inhibitors induce cell death selectively in cells that harbor activated kRasV12: the role of signal transducers and activators of transcription 1 and p21. Cancer Res 67: 8477-8485, 2007. 